\title{
COMPARISON OF BAYESIAN ANALYSIS AND PET-CT FINDINGS WITH RESPECT TO LIKELIHOOD OF MALIGNANCY IN SOLID AND SUBSOLID SOLITARY PULMONARY NODULES.
}

\footnotetext{
1. MBBS

Attending Physician

Department of Chest Diseases

Ankara Chest Diseases and Chest

Surgery Training and Research

Hospital, Ankara.

2. MBBS

Assistant Professor

Department of Emergency Medicine

Amasya University Sabuncuoglu

Serefeddin

Training and Research Hospital,

Amasya, Turkey

3. MBBS

Consultant

Department of Radiology

Ankara Koru Hospital, Ankara,

Turkey.

4. MBBS

Attending Physician

Department of Chest Diseases

Ankara Chest Diseases and Chest

Surgery Training and Research Hospital, Ankara.

5. MBBS

Attending Physician

Department of Chest Diseases

Ankara Chest Diseases and Chest

Surgery Training and Research

Hospital, Ankara.

6. MBBS

Associate Professor

Ankara Chest Diseases and Chest Surgery Training and Research

Hospital, Ankara.
}

Correspondence Address:

Dr. Sema Avci

Amasya University Sabuncuoglu

Serefeddin Training and Research

Hospital Amasya, Turkey.

dnzlsema@gmail.com

Article received on:

03/05/2019

Accepted for publication:

24/07/2019

\section{Oral Mentes' ${ }^{1}$, Sema Avci², Suzan Bahadır ${ }^{3}$, Meric Unver ${ }^{4}$, Murat Yildiz ${ }^{5}$, Feza Ugurman ${ }^{6}$}

ABSTRACT... Objectives: To determine whether Bayesian Analysis (BA) predicts malignancy with similar specificity and sensitivity values in both subgroups of solitary pulmonary nodules (SPNs) and to compare PET-CT findings in solid and subsolid subgroups of PET-CT scanned SPNs. Study Design: An observational study. Setting: Department of Chest Diseases, Ankara Chest Diseases and Chest Surgery Training and Research Hospital. Period: February 2013 to February 2016. Materials and Methods: 156 patients with SPNs and whose histopathological diagnosis confirmed by fiberoptic bronchoscopy biopsy, transthoracic tru-cut biopsy or surgical methods were evaluated retrospectively. BA and PET-CT findings of nodules were evaluated. Results: $73.3 \%$ of male patients and $80 \%$ of females with subsolid SPN were diagnosed malignant. BA was statistically significantly found to be consistent with definitive diagnosis in Kappa compliance analysis in solid and nonsolid nodules $(p<0.005$ kappa $=0.604 ; p=$ 0.023 kappa $=0.358$ ). The sensitivity of BA in solid and subsolid nodules was $63.6 \%$ and $80 \%$, respectively, while their specificity was $93.4 \%$ and $73 \%$, respectively. Positive predictive values (PPVs) were found to be $84 \%$ in solid nodules and $36 \%$ in subsolid nodules. Negative predictive values (NPVs) were calculated as $83 \%$ in solid nodules and $95 \%$ in subsolid nodules. In the patients with SPN included in our study, Kappa compliance analysis was performed separately in the solid and subsolid subgroups of patients who underwent PET-CT. When the cut-off value of Kappa compliance analysis in solid nodules was taken 2.5, definitive diagnosis and suvmax uptake were found to be statistically consistent $(p<0.005$ kappa $=0.638)$. When the cut-off value of Kappa compliance analysis in subsolid nodules was taken to be 2.5 as malignancy value, definitive diagnosis and suvmax uptake were found to be statistically consistent as in subgroup $(p=0.011$ kappa=0.399). When we took PET-CT suvmax cut-off value as 2.5 , sensitivity uptake and specificity of PET were found in solid nodules, to be $68.4 \%$ and $93.1 \%$, respectively. PPV was $86.7 \%$ and NPV was $82 \%$. The sensitivity and specificity values of subsolid subgroup were $70 \%$ and $75 \%$ respectively, whereas the PPVs and NPVs were $50 \%$ and $87.5 \%$, respectively. Conclusion: In subsolid SPNs, as in BA, PET-CT seems to be more reliable when used exclusively in malignancy exclusion. Although a significant suvmax cut-off value was determined for malignancy, the PPV of PET-CT is lower than that of solid SPNs.

Key words: $\quad$ Bayesian Analysis, PET-CT, Solitary Pulmonary Nodule,

Article Citation: Mentes O, Avci S, Bahadır S, Unver M, Yildiz M, Ugurman F. Comparison of Bayesian analysis and PET-CT findings with respect to likelihood of malignancy in solid and subsolid solitary pulmonary nodules. Professional Med J 2019; 26(11):1971-1982. DOI: 10.29309/TPMJ/2019.26.11.3674

\section{INTRODUCTION}

A solid pulmonary nodul (SPN) is a homogeneous soft-tissue attenuation at computed tomography (CT) that covers the underlying vascular and bronchial structures and which is identified as round or irregular opacity with a mean axial diameter of 0.3-3.0 cm. ${ }^{1}$ A subsolid pulmonary nodule is a pure ground-glass or partially solid attenuation at CT that does not cover entirely the underlying bronchial and vascular structures which is identified as round or irregular opacity with a mean axial diameter of $0.3-3.0 \mathrm{~cm} .{ }^{1}$ For the first time, in 1986, Cummings et al., used Bayesian theorem in determining the risk of malignancy in solitary pulmonary nodules. In order to determine the likelihood of malignancy, they used 4 parameters i.e. age, smoking, lesion diameter, and the malignancy prevalency of solitary pulmonary nodules in the population. ${ }^{2}$ 
The first recognized clinical application of PETCT was in the field of investigation of solitary pulmonary nodules and lung cancers. PET is a imaging method that works on the basis of crystal detection of two opposite gamma rays resulting from collision of electrons in the tissue with positrons emitted from radionuclides such as fluorodeoxyglucose (FDG). While PET collects information about the body's function with radiopharmaceuticals, CT provides anatomical details of normal and pathological tissues in the body. The data obtained by combining both devices are in the form of fusion images obtained by overlapping PET images, CT images and the both on the workstation. Thus, the increase in intracellular enzymes (hexokinase and phosphofructokinase) which provide glycolysis in tumor cells, and the contrasting lowering rate of dephosphorylation due to the decrease in glucose-6-phosphatase enzyme activity resulting in more glucose consumption than normal cells is the basis of PET imaging by using FDG. ${ }^{3,4}$

PET-CT has a sensitivity of $96.8 \%$ and a specificity of $77 \%$ in the differential diagnosis of benignmalignant SPN. However, in some types of adenocarcinoma, false negativity in carcinoid and tumors smaller than $1 \mathrm{~cm}$ may be observed, false positivity may be seen in infectious and inflammatory conditions. ${ }^{5,6}$ PET-CT is useful for the differentiation of benign and malignant nodules up to $1 \mathrm{~cm}$. For nodules below 8-10 $\mathrm{mm}$, evaluation with PET-CT should be avoided. In addition, tumors with low metabolic activity (eg, adenocarcinoma in situ, carcinoid, etc.) may cause false negative results. Considering the other causes of false negativity, preoperative biopsy should be performed in patients with high risk even if FDG-PET is negative. False positivity may also be seen in SPN and mediastinum in tuberculosis, endemic mycosis, sarcoidosis and some other infectious or inflammatory diseases. Therefore, biopsy materials remain to be the gold standard for the final diagnosis of SPNs., ${ }^{7,8}$ The aim of this study was to determine whether Bayesian analysis predicts malignancy with similar specificity and sensitivity values in both subgroups of solitary pulmonary nodules and to compare PET findings in solid and subsolid subgroups of PET-CT-scanned solitary pulmonary nodules.

\section{BAYESIAN ANALYSIS (PROBABILITY OF THE MALIGNANCY)}

By evaluating clinical and radiological data obtained from the patient, the likelihood ratio (LR) and malignancy expectation (pCA) are calculated. These calculations are made by a method developed by the English mathematician Bayes. Accordingly, the likelihood ratio is calculated with the formula;

$\mathrm{LR}=$ Number of malignant single lung nodules with finding a / Number of benign single lung nodules with finding $a$. The fact that LR is 1.0 indicates a $50 \%$ probability, benign if less than 1.0 , and a malignant single lung nodule if greater than 1.0. Based on these characteristics, the odds ratio (probability ratio) is calculated for malignancy. The local malignancy prevalence (LRprev) of the population should be known in this calculation. Based on this, OddsCA (cancer possibility) is found. LRprev $=$ prevalence of malignancy / 1- prevalence of malignancy OddsCA $=$ LRprev $\times$ LRa $\times$ LRb $\times$ LRc $\times \ldots$ PCA (malignancy expectancy) is also calculated by using the obtained OddsCA. $\mathrm{pCA}=$ OddsCA $/ 1+$ OddsCA

\section{METHODOLOGY}

After obtaining the necessary ethics committee approval for the study, 156 patients with solitary pulmonary nodules and whose histopathological diagnosis confirmed by FOB (Fiberoptic Bronchoscopy) biopsy, transthoracic tru-cut biopsy or surgical methods were evaluated retrospectively. The patients had been followed up and treated in Ankara Chest Diseases and Chest Surgery Training and Research Hospital between February 2013 and February 2016. Radiological measurements and characteristics of nodules are defined by the recommendations of "Fleischner Society". 9,10 "Bayesian Analysis" consisting of 6 radiological and 4 clinical parameters was used to determine the risk of malignancy of the patients alongside with "SPN Calculator" available free of charge on the www.chestx-ray.com website. ${ }^{11,12,13}$ Age, smoking, hemoptysis and presence of 
previous extrathoracic malignancy in patiens were evaluated. Nodules' diameter, growth rate, localization, contour, calcification pattern, wall thickness for cavitary nodules were evaluated. Also, the densities of the nodules were calculated as "Hounsfield Unit" (HU). The suvmax of the nodules of patients scanned with PET-CT were calculated and included in the malignancy risk analysis. Inclusion criteria was that the nodule be single, its diameter be more than $5 \mathrm{~mm}$, at least two-thirds of it be surrounded by lung parenchyma, not accompanied by mediastinal lymph node in pathological dimension, presence of no nodular atelectasis or obstructive pneumonia, the absence of nodule-associated pleural fluid, the patient previously be not diagnosed with lung cancer, malignancy of patients with extrathoracic malignancy be diagnosed more than 5 years ago and the definitive histopathologic diagnosis of nodules be made. Thorax CTs of the patients included in the study were examined in thin section $(1 \mathrm{~mm})$ thickness as recommended by "Fleischner Society. The false frosted glass image that could occur in thick sections was excluded. In this respect, it was decided that the nodules were solid or subsolid (Figure-1a-1d). 1a. Frosted glass- characterised nodule in right upper lobe anterolateral 1b. Subsolid nodule due to the frosted glass component on the posterolateral 1c. Solid nodules containing scattered air images $1 d$. The nodule that we evaluated in solid class, with a halo sign around it, which is probably the result of bleeding or edema. The diameters of the nodules were measured in 2 dimensions in the section where the nodule was the largest in the transverse CT sections. The arithmetic mean of the two measured values was determined as the nodule diameter. The growth rates of nodules were calculated only by thoracic CTs taken in patients with nodule follow-up or in another center by appropriate technique. Because the volume of the sphere is 4 pi $r 3 / 3$, the time during which an increase of about $26 \%$ in diameter of the nodule occured was determined as volume doubling time. The duration of doubling time, if between 20-400 days, was evaluated as the rate of malignant growth, and if less than 20 days or more than 400 days, evaluated as benign growth rate. ${ }^{14,15}$ The edge properties of the nodules were evaluated in three categories: smooth, lobular or irregular and spiculated contours. Calcification patterns of nodules were evaluated in thorax CT. Popcorn-shaped calcification, stratified like onion skin, central and diffuse homogeneous calcification patterns inside the nodule were evaluated as benign calcification.

Mediastinum cross-sections of CTs were also evaluated after thoracic CT scan of the patients were categorized into solid and subsolid. The densities of the nodules seen in the mediastinal sections were calculated over multiple locations of the nodule and and the average value was taken. Parenchymal sections of nodules that could not be seen in the mediastinal sections were examined. Multiple measurements were made on the most solid appearing hyperdense areas of the nodules and the average value was taken. The measured HU values were included in the malignancy risk analysis and the values above $15 \mathrm{HU}$ were interpreted as malignancy.

While PET-CTs of patients with solitary pulmonary nodes were included in Bayes' risk analysis, 2.5 suvmax was determined as cutoff value for malignancy. The values above 2.5 , which the PET-CT predicted as malignant and the values below 2.5, which were predicted as benign by PET-CT, were used to measure the value of PETCT in detecting the risk of malignancy both in Bayesian analysis and alone. After all radiological measurements and clinical evaluations of the patients were completed, Bayesian analysis was performed by means of sonra SPN Calculator. As a result, the malignancy risks of a certain percentage of the patients were calculated. The general approach is to follow patients at risk of less than $5 \%$, to operate over $60 \%$, while a biopsy is recommended for between $5 \%$ and $60 \%$. To use these values in statistical analysis, Bayesian analysis was evaluated as benign under $5 \%$, as malignant over $60 \%$ and as ambigous for values between $5 \%$ and $60 \%$.

\section{Statistical Analysis}

The data obtained in this study were analyzed by SPSS 22.0 package program. ROC analysis, Kappa compliance analysis, and Mann-Whitney 
$\mathrm{U}$ tests were used to analyze the data. 0.05 was determined as significance level, if $p<0.05$ there is a significant difference, if $p>0.05$ there is no significant difference.

\section{RESULTS}

$43(27.5 \%)$ of the patients were female and $113(72.5 \%)$ were male. The mean age of the patients was 59.8 years. $60.2 \%$ of male patients and $51.5 \%$ of female patients with solid SPN had malign histopathology. $73.3 \%$ of male patients and $80 \%$ of females with subsolid SPN were diagnosed malignant. A total of 138 patients were diagnosed surgically, 13 patients diagnosed through transthoracic tru-cut biopsy and 5 patients diagnosed through FOB biopsy. In solid and subsolid groups, the relationship between age and malignancy was significantly increased in patients with solid SPN $(p=0.001)$, whereas in elderly group the older patients received more benign diagnosis. This result in the subsolid group was not statistically significant $(p=0.302)$. When the patients included in the study were divided into two groups as solid and subsolid, the risk of malignancy increased as the nodule diameters increased. While this relationship was significant in solid nodules $(p=$ 0.031 ), it was statistically insignificant in subsolid nodules $(p=0.101)$. When the cutoff values of the nodule diameters in both groups were examined by ROC analysis in terms of malignancy, it was calculated as $19.5 \mathrm{~mm}$ in solid nodules and $14.5 \mathrm{~mm}$ in subsolids. However, the area under the curve in subsolid group was not statistically significant. Of the 156 nodules, 84 were in the right lung and 72 in the left lung. Of these, 76 were located in the upper lobe, 75 in the lower lobe and 5 in the middle lobe. Of the nodules located in the upper lobe, 48 were malignant histopathology. 46 of the lower lobe nodules were malignant. Of the middle lobe nodules, 3 were malignant. There was no significant difference between the location of nodules and malignancy $(p>$ 0.05). Densities of the nodules of patients were calculated in terms of Hounsfield Unit based on the thin-sectioned thorax computed tomographs suitable for technical measuring. ROC analysis was performed to determine a significant cutoff value for malignancy. $28.5 \mathrm{HU}$ was calculated as cut off value for malignancy. However, the area under the curve was not statistically significant $(p>0.05)$. When the histopathological diagnosis of the patients included in the study were divided into 2 subgroups as malignant and benign, malignant diagnosis were adenocarcinoma in 55 patients, primary lung squamous cell carcinoma in 17 patients. The diagnosis of 3 patients was adenosquamous cell carcinoma. Five of the patients were diagnosed as metastatic nodules. Metastases were renal cell carcinoma, breast carcinoma, follicular thyroid carcinoma, endometrial stromal sarcoma metastasis and trophoblastic tumor metastasis. One patient was diagnosed as large cell carcinoma and 2 patients were diagnosed as small cell carcinoma. Schwannoma and malignant neuroendocrine tumors were diagnosed in two different patients. Of the 5 patients with carcinoid tumor histopathology, 3 had typical carcinoid and 2 had atypical carcinoid. As lower-grade malignant formations, one patient was diagnosed with solitary fibrous tumor, one patient with epithelioid hemangioendothelioma and 2 patients with sclerosing hemangioma. One of our patients was diagnosed with atypical adenomatous hyperplasia as a precancerous lesion. In our benign patient subgroup, 21 patients were diagnosed with hamartoma.

Histopathology of 7 patients was reported as anthracotic lung parenchyma. Granulomatous inflammation in 5 patients, organized pneumonia in 5 patients, fibrotic changes in 3 patients, bronchiolitis in 2 patients and ischemic necrosis and abscess diagnosis in two different patients were present. 2 patients had intraparenchymal lymphnode, 2 patients had dystrophic calcification, 1 patient had rheumatoid nodule, 1 patient had silicotic nodule and 1 patient had interstitial pneumonia and endothelial proliferation, of which significance could not be determined. Kappa compliance analysis was performed in the way that the Bayesian analysis results obtained from the clinical and radiological parameters of the patients included in the materials and methods of the study were compared with the definitive histopathological diagnosis of solid and sub solid patients. Bayesian analysis was statistically 
significantly found to be consistent with definitive diagnosis in Kappa compliance analysis in solid nodules $(p<0.005$ kappa $=0.604)$ (Table-I). Kappa compliance analysis performed with definite diagnosis and Bayesian analysis in solid nodules Bayesian analysis was statistically significantly found to be consistent with definitive diagnosis in Kappa compliance analysis in non solid nodules as well ( $p=0.023 \mathrm{kappa}=0.358$ ) (Table-II). Kappa compliance analysis performed with definite diagnosis and Bayesian analysis in subsolid nodules. The sensitivity of Bayes analysis in solid and subsolid nodules was $63.6 \%$ and $80 \%$, respectively, while their specificity was $93.4 \%$ and $73 \%$, respectively. Positive predictive values were found to be $84 \%$ in solid nodules and $36 \%$ in subsolid nodules. Negative predictive values were calculated as $83 \%$ in solid nodules and $95 \%$ in subsolid nodules. In the patients with SPN included in our study, Kappa compliance analysis was performed separately in the solid and subsolid subgroups of patients who underwent PET-CT. When the cut-off value of Kappa compliance analysis in solid nodules was taken 2.5, definitive diagnosis and suvmax uptake were found to be statistically consistent $(p<0.005$ kappa $=0.638$ ) (Table-III). Kappa compliance analysis between PET-CT suvmax and definitive diagnosis of solid nodules. When the cut-off value of Kappa compliance analysis in subsolid nodules was taken to be 2,5 as malignancy value, definitive diagnosis and suvmax uptake were found to be statistically consistent as in subgroup $(p=0.011$ kappa $=0.399) \quad$ (Table-IV). Kappa compliance analysis between PET-CT suvmax and definitive diagnosis of subsolid nodules.

\begin{tabular}{|c|c|c|c|c|c|}
\hline \multicolumn{6}{|c|}{ Bayesian Analysis Results } \\
\hline \multirow{2}{*}{ Solid } & \multicolumn{2}{|c|}{ Definitive Histopathologic Diagnosis } & \multirow{2}{*}{ Total } & \multirow{2}{*}{ Kappa } & \multirow{2}{*}{$\mathbf{P}$} \\
\hline & Benign & Malign & & & \\
\hline \multirow{3}{*}{ Benign } & 21 & 4 & 25 & \multirow{9}{*}{0.604} & \multirow{9}{*}{0.000} \\
\hline & $84.0 \%$ & $16.0 \%$ & $100.0 \%$ & & \\
\hline & $63.6 \%$ & $6.6 \%$ & $26.6 \%$ & & \\
\hline \multirow{3}{*}{ Malign } & 12 & 57 & 69 & & \\
\hline & $17.4 \%$ & $82.6 \%$ & $100.0 \%$ & & \\
\hline & $36.4 \%$ & $93.4 \%$ & $73.4 \%$ & & \\
\hline \multirow{3}{*}{ Total } & 33 & 61 & 94 & & \\
\hline & $35.1 \%$ & $64.9 \%$ & $100.0 \%$ & & \\
\hline & $100.0 \%$ & $100.0 \%$ & $100.0 \%$ & & \\
\hline
\end{tabular}

Table-I. Kappa compliance analysis performed with definite diagnosis and Bayesian analysis in solid nodules

\begin{tabular}{|c|c|c|c|c|c|}
\hline \multicolumn{6}{|c|}{ Bayesian Analysis Results } \\
\hline \multirow{2}{*}{ Subsolid } & \multicolumn{2}{|c|}{ Definitive Histopathologic Diagnosis } & \multirow{2}{*}{ Total } & \multirow{2}{*}{ Kappa } & \multirow{2}{*}{$\mathbf{P}$} \\
\hline & Benign & Malign & & & \\
\hline \multirow{3}{*}{ Benign } & 4 & 7 & 11 & \multirow{9}{*}{0.358} & \multirow{9}{*}{0.023} \\
\hline & $36.4 \%$ & $63.6 \%$ & $100.0 \%$ & & \\
\hline & $80.0 \%$ & $26.9 \%$ & $35.5 \%$ & & \\
\hline \multirow{3}{*}{ Malign } & 1 & 19 & 20 & & \\
\hline & $5.0 \%$ & $95.0 \%$ & $100.0 \%$ & & \\
\hline & $20.0 \%$ & $73.1 \%$ & $64.5 \%$ & & \\
\hline \multirow{3}{*}{ Total } & 5 & 26 & 31 & & \\
\hline & $16.1 \%$ & $83.9 \%$ & $100.0 \%$ & & \\
\hline & $100.0 \%$ & $100.0 \%$ & $100.0 \%$ & & \\
\hline
\end{tabular}

Table-II. Kappa compliance analysis performed with definite diagnosis and Bayesian analysis in subsolid nodules 


\begin{tabular}{|c|c|c|c|c|c|}
\hline \multicolumn{6}{|c|}{ PET-CT Suvmax Values } \\
\hline \multirow{2}{*}{ Solid } & \multicolumn{2}{|c|}{ Definitive Histopathologic Diagnosis } & \multirow{2}{*}{ Total } & \multirow{2}{*}{ Kappa } & \multirow{2}{*}{$\mathbf{P}$} \\
\hline & Benign & Malign & & & \\
\hline \multirow{3}{*}{ Benign } & 26 & 4 & 30 & \multirow{9}{*}{0.638} & \multirow{9}{*}{0.000} \\
\hline & $86.7 \%$ & $13.3 \%$ & $100.0 \%$ & & \\
\hline & $68.4 \%$ & $6.9 \%$ & $31.3 \%$ & & \\
\hline \multirow{3}{*}{ Malign } & 12 & 54 & 66 & & \\
\hline & $18.2 \%$ & $81.8 \%$ & $100.0 \%$ & & \\
\hline & $31.6 \%$ & $93.1 \%$ & $68.8 \%$ & & \\
\hline \multirow{3}{*}{ Total } & 38 & 58 & 96 & & \\
\hline & $39.6 \%$ & $60.4 \%$ & $100.0 \%$ & & \\
\hline & $100.0 \%$ & $100.0 \%$ & $100.0 \%$ & & \\
\hline
\end{tabular}

Table-III. Kappa compliance analysis between PET-CT suvmax and definitive diagnosis of solid nodules

\begin{tabular}{|c|c|c|c|c|c|}
\hline \multicolumn{6}{|c|}{ PET-CT Suvmax Values } \\
\hline \multirow{2}{*}{ Subsolid } & \multicolumn{2}{|c|}{ Definitive Histopathologic Diagnosis } & \multirow{2}{*}{ Total } & \multirow{2}{*}{ Kappa } & \multirow{2}{*}{$\mathbf{P}$} \\
\hline & Benign & Malign & & & \\
\hline \multirow{3}{*}{ Benign } & 7 & 7 & 14 & \multirow{9}{*}{0.399} & \multirow{9}{*}{0.011} \\
\hline & $50.0 \%$ & $50.0 \%$ & $100.0 \%$ & & \\
\hline & $70.0 \%$ & $25.0 \%$ & $36.8 \%$ & & \\
\hline \multirow{3}{*}{ Malign } & 3 & 21 & 24 & & \\
\hline & $12.5 \%$ & $87.5 \%$ & $100.0 \%$ & & \\
\hline & $30.0 \%$ & $75.0 \%$ & $63.2 \%$ & & \\
\hline \multirow{3}{*}{ Total } & 10 & 28 & 38 & & \\
\hline & $26.3 \%$ & $73.7 \%$ & $100.0 \%$ & & \\
\hline & $100.0 \%$ & $100.0 \%$ & $100.0 \%$ & & \\
\hline
\end{tabular}

When we took PET-CT suvmax cut-off value as 2.5 , sensitivity uptake and specificity of PET are found in solid nodules, to be $68.4 \%$ and $93.1 \%$, respectively. Positive predictive value was $6.7 \%$ and negative predictive value was $82 \%$. The sensitivity and specificity values of subsolid subgroup were $70 \%$ and $75 \%$ respectively, whereas the positive predictive and negative predictive values were $50 \%$ and $87.5 \%$, respectively. When we divided the patients included in the study into solid and subsolid subgroups, the cut-off value of solid nodules was found to be 3.01 when we separately performed ROC analysis for PETBT suvmax uptake in terms of malignancy. The area under the curve appears to be statistically significant ( $p=0.0001$ ) (Figure-2). ROC analysis of PET-CT uptake in solid nodules for malignancy.

In the subsolid subgroup the cut-off value was obtained as 2.73. The area under the curve is statistically significant $(p=0.006)$.

(Figure-3). ROC analysis of PET-CT uptake in subsolid nodules for malignancy.

Figure-1a-1d.

1a. Frosted glass- characterised nodule in right upper lobe anterolateral.

1b. Subsolid nodule due to the frosted glass component on the posterolateral.

1c. Solid nodules containing scattered air images.

1d. The nodule that we evaluated in solid class, with a halo sign around it, which is probably the result of bleeding or edema. 


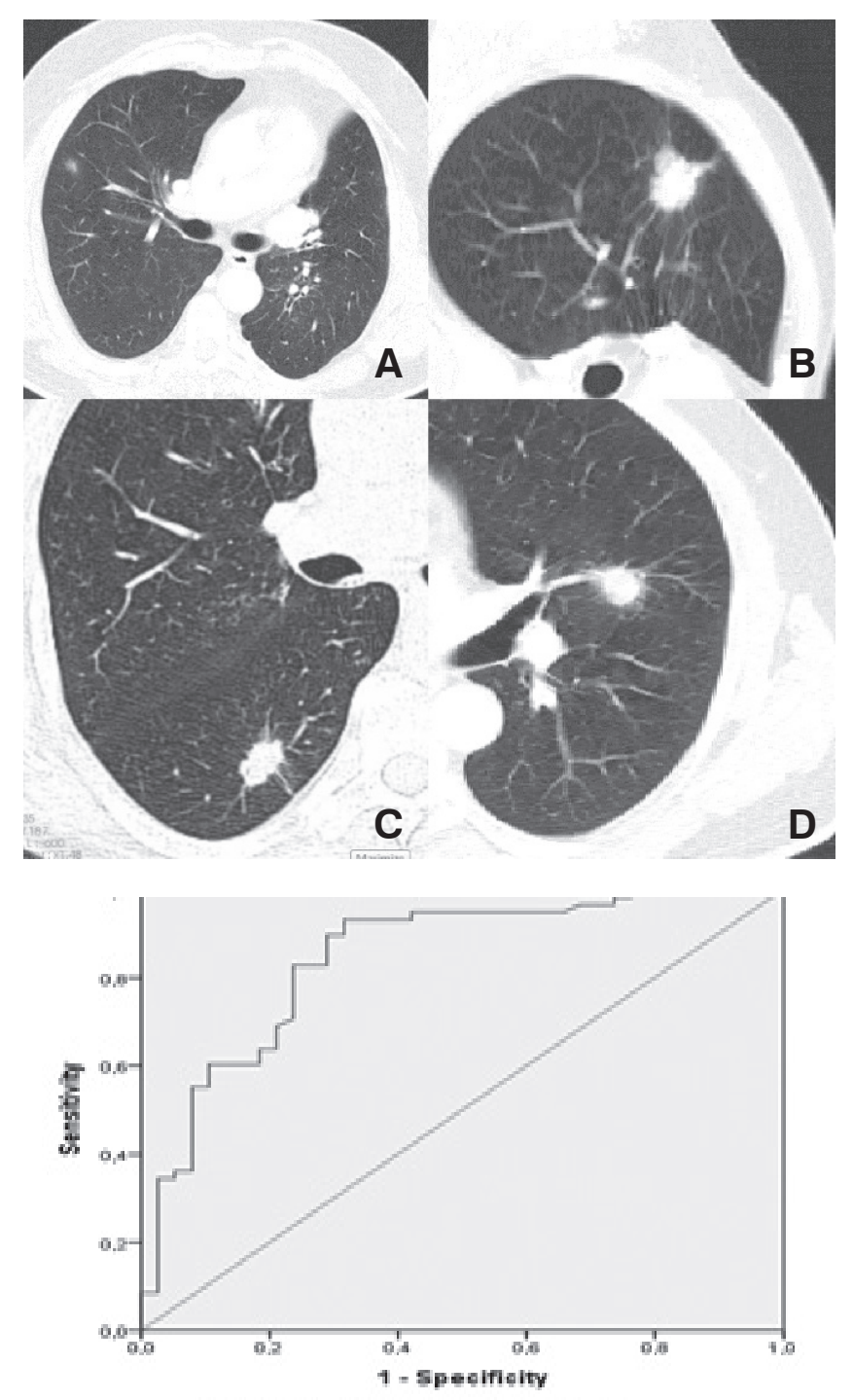

Diagonal segments are produced by ties.

Figure-2. ROC analysis of PET-CT uptake in solid nodules for malignancy

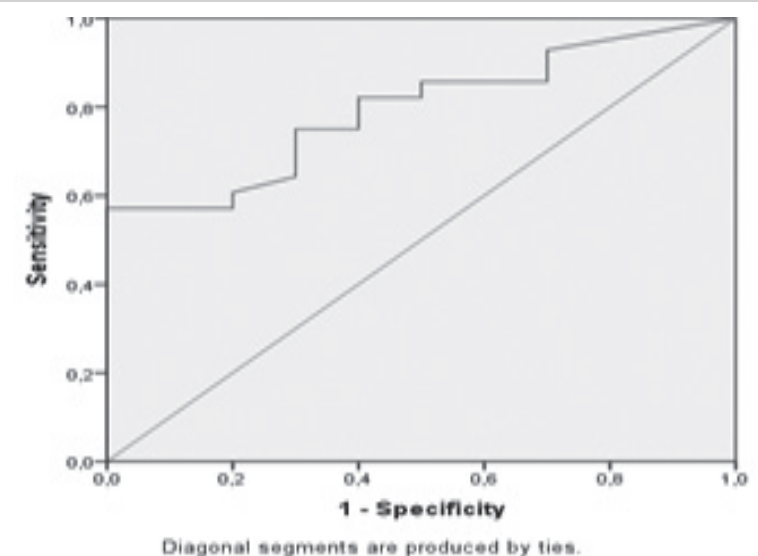

Figure-3. ROC analysis of PET-CT uptake in subsolid nodules for malignancy

\section{DISCUSSION}

In the United States, 150.000 new pulmonary nodules are diagnosed annually. According to the National Lung Screening Program published in 2011, the use of CT for screening purposes increases this rate. ${ }^{16,17}$

Gurney et al. demonstrated that for the solitary pulmonary nodules, they can be combined with Bayesian analysis by using the likelihood ratios determined in the literature. They developed a theory using 15 radiological parameters and 4 clinical parameters among the 15 malignancy findings and 19 benign findings in the literature and, they applied it on 66 patients in another study. They determined the most important radiological risks for malignant nodules as wall thickness, spicular contour and lesion size for cavitary lesions and as benign character growth rate and benign character calcification for benign nodules. ${ }^{11}$ In the study of Swensen et al., it was aimed to determine the independent malignancy predictors in SPNs. Various clinical and radiological parameters, clinically, age, sex, smoking, extrathoracic malignancy history, asbestos exposure, presence of interstitial lung disease, presence of chronic obstructive pulmonary disease and radiologically, lesion diameter, localization, contour, cavitary wall diameter and presence and shape of calcification, were studied in $2 / 3$ of the patients, were tested on $1 / 3$ of them. Age, smoking and cancer history were independent clinical malignancy predictors, while lesion diameter, upper lobe localization and spicule contour were independent radiological predictors. ${ }^{12}$

In the study of Soardi et al., a new Bayesian calculator was aimed for solid solitary pulmonary nodules and radiological and clinical risks were updated for benign malignant differentiation. According to this study, Bayesian analysis is very successful in characterizing solitary pulmonary nodules, it is quite successful in predicting the volume doubling time-VDT of malignancy. VDT between 25 and 400 days is a risk for malignancy. In addition, VDT, nodule size, morphology, density were found to be the best malignancy predictors. ${ }^{18}$ This calculator, which is thought to 
be more specific and sensitive for solid solitary pulmonary nodules, including Bayesian analysis, gave us the idea that in the first detection of nodules, the nodule should be determined to be solid or subsolid (pure frosted glass and semisolid nodules. ${ }^{19}$ In accordance with this information in the literature, patients with malignant subsolid nodules are more likely to be younger, nonsmoker and to have radiologically lower-density nodules. In our study, adenocarcinoma was diagnosed in 28 of 40 subsolid SPNs and we had a patient with subsolid nodules with atypical adenomatous hyperplasia. Therefore, when SPN is detected in one patient, If we want to calculate the risk of malignancy in the initial diagnosis, we should consider that there are some differences in risk factors in solid and subsolid SPNs. In our study, subsolid patient group consisted of 40 people and solid group consisted of 116 people. Bayesian analysis was performed for all patients. But all the risk factors of all patients could not be reached. The Bayesian Analysis was performed only with accessible risk factors of the patients. Risk factors reached in patients number large enough to be studied statistically were studied separately from the Bayesian Analysis. These were compared statistically in the subgroups of solid and subsolid patients. The average age of men in our study, as stated in the findings, was higher than the female population. Our male population was higher in both subgroups. While the percentage of malignancy in males was higher in the solid subgroup, the percentage of malignancy in females was higher in subsolid subgroup.

The highest prevalence of malignancy was $80 \%$ and it belonged to women with subsolid SPN. The lowest prevalence of malignancy was 51.5\% and belonged to women with solid SPN. The malignancy rate in our entire male population was higher than the one in our entire female population. In solid SPNs, as age increases, malignancy significantly increases ( $p<0.05$ ). However, as age increases in subsolid SPNs, the likelihood of malignant diagnosis of the nodule decreases. Since the number of our subsolid SPN patient population was small, this data was not statistically significant $(p>0.05)$. When the diameter of all SPNs were measured, as the nodule diameter increased in the solid subgroup, the malignancy rate increased significantly, while the subsolid subgroup showed an increase in the malignancy rate as the nodule diameter increased but this was not statistically significant and this was attributed to the low number of samples in subsolid group $(p>0.05)$. While in the literature, it was reported that malignancy was significantly increased after $20 \mathrm{~mm}$ nodule diameter for all SPNs. ${ }^{16,20}$ In our study, the cut-off value calculated for solid SPNs was found to be $19.5 \mathrm{~mm}$. This data supports the literature. The cut-off value for the subsolid SPNs was calculated as $14.5 \mathrm{~mm}(p>0.05)$. The fact that this data was not statistically significant was attributed to the insufficient number of subsolid SPNs. There was no significant difference between the location of the nodules of the patients involved in the study and the risk of malignancy in the lung ( $p>0.05$ ). When we look at the literature, it is said that the nodules located in the upper lobe are more malignant and this is attributed to the easier access of the inhaled carcinogens to the upper lobes. ${ }^{14,21}$

CT density of 59 patients from solid SPNs and 30 patients from subsolid SPNs were able to be measured in the $\mathrm{HU}$. The mean value for malignant nodules was $36.7 \mathrm{HU}$ in the solid subgroup and $30.8 \mathrm{HU}$ in benign nodules when analyzed separately for malignancy. The mean value of malignant nodules was $-29.4 \mathrm{HU}$ in subsolid subgroups and $-97.4 \mathrm{HU}$ for benign nodules. However, the density of CT was not significant in terms of malignancy in niether solid or subsolid subgroups ( $p>0.05$ ). ROC analysis was performed for all nodules in relation to malignancy of the $\mathrm{HU}$ on CT. In terms of malignancy, cutoff value was calculated as 28,5 $\mathrm{HU}$. However, the area under the curve was not statistically significant $(p>0.05)$. When we look at the literature, it is seen that the values above $15 \mathrm{HU}$ are found to be significant in terms of malignancy. ${ }^{21}$ When we look at the subsolid malignant subgroup of our study, we see that the average value of the $\mathrm{HU}$ is even below zero. In addition, almost half of the solid subgroup could not be reliably measured due to technical 
reasons. A significant cut-off value was not found since the number of solid and subsolid samples whose density was measurable was close to each other and the difference between the subsolid malignant and the average HU values of the solid malignancies was high. Nevertheless, as the HU levels increased in both the solid and subsolid subgroups, the malignancy increased ( $p>0.05)$. In the study titled 'Road Map in Lung Cancer "published in February 2016 with the contributions of Turkish Thoracic Society, while the most common primary lung cancer in our country was identified as a subcellular non-squamous cell carcinoma subtype, when the histopathology of the patients included in our study was examined; adenocarcinoma was the most malignant histopathology. The most common benign histopathology was hamartoma, supporting the literature. ${ }^{22}$ Based on the data that constitute the basis of our study, Bayesian analysis data; kappa compliance analyzes were significant in both the solid and subsolid subgroups ( $p<0.05)$. In other words, the Bayesian analysis data and therefore the likelihood ratios, which are the basis of the SPN. ${ }^{11,12}$ Calculator application, which can be accessed free of charge at www.chestx-ray.com, at first sight, seem to be successful in detecting the likelihood of malignancy in the initial detection of SPN for both solid and subsolid nodules. When we compare the solid and subsolid subgroups, however, we see that $p$ value is lower $(p=0,000)$ for solid subgroup. The positive and negative predictive values of Bayesian analysis in solid nodules were similarly found to be $84 \%$ and $83 \%$, respectively, and $36 \%$ and $95 \%$, respectively. In other words, when we detect a subsolid SPN, If the value obtained by calculating the probability of malignancy by Bayesian analysis is below $5 \%$, this nodule is not malignant with a probability of $95 \%$. But if the value obtained as a result of Bayesian analysis is over $60 \%$, the probability of being malignant for this subsolid nodule is as low as $36 \%$. In this case, although Bayesian analysis in subsolid SPNs seems to be statistically compatible in predicting malignancy, it is statistically more reliable to use Bayesian analysis only for malignancy exclusion in subsolid SPNs. For solid SPNs, Bayesian analysis was found to be successful in both predicting and excluding malignancy. The reason for this is thought to be the fact that the likelihood ratios and 10-parameter Bayesian analysis data are included in the study without classifying nodules as solid and subsolid. However, patients with subsolid malignant SPNs can be younger, non-smoker or light smoker because of the clinical and radiological features of adenocarcinoma; radiologically, the nodules may have lower densities and a much longer doubling time and can be found in the upper lobe or lower lobe regardless of the exposure of the carcinogens. Also, PET-CT uptake may be lower. In the literature, likelihood ratios and Bayesian analysis data calculated exclusively for subsolid SPN does not exist. The Bayesian analysis data in the current literature seem to be quite successful, considering only benign causes in subsolid SPNs. However, since most of the malignant causes in subsolid SPNs are adenocarcinomas (In our study, 29 of 40 subsolid nodules were diagnosed as malignant and 28 of them were adenocarcinoma and 27 of 28 were primary lung adenocarcinoma) Bayesian analysis is poor in predicting malignancy in subsolid SPNs.

Because we had expected to get close results when planning our study, We planned to study the different likelihood rates for malignancy for subsolid SPNs and for different radiological or clinical risk factors (eg. solid/ frosted glass ratio ) for Bayesian analysis. However, We only reached too few subsolid SPNs to be statistically meaningful. In this respect, this thesis can be the first step to a larger study. When we look at the PET-CT findings which is also a basis for our study, we see similar results to Bayesian analysis. When PET-BT suvmax cut-off value for malignancy was taken as 2.5, Kappa compliance analysis between both solid and subsolid SPNs and PET-BT suvmax uptake is significant $(p<0.05)$. However, as in Bayesian analysis, the negative predictive value for subsolid SPNs is higher than the positive predictive value (NPV: $87.5 \%$ PPV: $50 \%$ ). On the other hand, the negative predictive value was found to be $82 \%$ and the positive predictive value was $86.7 \%$ in solid SPNs. As reported by Chun et al. In the literature, paradoxically, suvmax value in subsolid nodules is higher in inflammatory nodules compared to malignant nodules. Again, 
in the study of Tsushima et al., values above 1.5 SUVs (standardized uptake value) suggest benign pathology in SPNs which are subsolid on PET-CT. This data makes the value and the status of PET-CT controversial in subsolid nodules. ${ }^{23}$ In our study however, uptakes below 2,5 in subsolid SPNs suggest a significant benign pathology. However, the detection rate for malignancy remains above $50 \%$ in uptake above 2,5 . In subsolid SPNs, as in Bayesian analysis, PET-CT seems to be more reliable when used exclusively in malignancy exclusion. ROC analysis, made separately in solid and subsolid SPNs for PETCT suvmax in respect to malignancy, showed significant results. The cut-off value for the solid subgroup was 3.01 ( $p=0.0001$ ) and the cut-off value for subsolid subgroup was $2.73(p=0.006)$. In the initial detection of subsolid SPNs, while the decision to follow subsolid SPNs is safely possible with PET-BT and Bayesian analysis, it is not correct to decide on an operation with a preliminary diagnosis of malignancy in order to avoid morbidity and mortality.

Also, considering that the vast majority of subsolid SPNs are caused by adenocarcinomas, due to longer doubling times compared to other malignancies it is easier for the physician to take the decision "to follow the patient." However, as already mentioned, when patients are informed of "a stain" in the lung, they associate it with lung cancer. ${ }^{17}$ Following a patient in that mood for 3-4 years will not be very easy neither for the physician nor for the patient. For these reasons, new methods are needed to reliably calculate the likelihood of malignancy, especially at the initial detection of subsolid SPNs. Bayesian analysis seems useful in this regard, but new risk factors for subsolid nodules should be defined and likelihood rates should be calculated in large patient groups and as Gurney did in all nodules, it should be confirmed with a second study on a different patient population. Although the biopsy is recommended for those who are estimated to be between $5 \%$ and $60 \%$ in Bayesian malignancy risk analysis, the location and size of the nodule from a technical point of view and the experience of the practitioner sometimes complicate the biopsy. Possible positive results in terms of malignancy will often lead us to curative surgery. Negative results, on the other hand, can not convince the physician who followed the patient that the nodule is benign, and the nodule continues to be monitored. While these situations are a completely separate subject of research including physicians and patients, they are also clinical experiences that should be taken into consideration. The findings in the literature regarding the PET-CT findings of subsolid SPNs are controversial. In our study, although a significant suvmax cut-off value was determined for malignancy, the positive predictive value of PET-CT is lower than that of solid SPNs. For this reason, when we detected a subsolid SPN with suvmax uptake above 2.5 , it is not reliable to evaluate this nodule to be malignant as did in the solid subgroup.

\section{CONCLUSION}

In subsolid SPNs, as in BA, PET-CT seems to be more reliable when used exclusively in malignancy exclusion. Although a significant suvmax cut-off value was determined for malignancy, the PPV of PET-CT is lower than that of solid SPNs.

Copyright@ 24 July, 2019.

\section{REFERENCES}

1. Ridge CA, Yildirim A, Boiselle PM, Franquet T, SchaeferProkop CM, Tack D, et al. Differentiating between Subsolid and Solid Pulmonary Nodules at CT: Interand Intraobserver Agreement between Experienced Thoracic Radiologists. Radiology 2016; 278 (3):88896.

2. Cummings SR, Lillington GA, Richard RJ. Estimating the probability of malignancy in solitary pulmonary nodules: a Bayesian approach. Am Rev Respir Dis 1986; 134: 449-52.

3. Mudun A. Türkiye'de PET görüntülemenin durumu. Turk J Nucl Med 2008; 17:1-9.

4. Weber G, Cantero A. Glucose-6-phosphatase activity in normal, pre-cancerous, and neoplastic tissues. Cancer Res 1955; 15:105-8.

5. Sharma P, Singh H, Basul S, Kumar R. Positron emission tomography computed tomography in the management of lung cancer: An update. South Asian J Cancer 2013; 3: 171-8. 
6. Sim YT, Poon FW. Imaging of solitary pulmonary nodüle-a clinical review. Quant Imaging Med Surg 2013; 3: 316-26.

7. Herder GJ, Golding RP, Hoekstra OS, Comans EF, Teule GJ, Postmus PE, et al. The performance of (18) F-fluorodeoxyglucose positron emission tomography in small solitary pulmonary nodules. Eur J Nucl Med Mol Imaging 2004; 31(9):1231-6.

8. Cheran SK, Nielsen ND, Patz JrEF. False-negative findings for primary lung tumors on FDG positron emission tomography: staging and prognostic implications. Am J Roentgenol 2004; 182(5):1129-32.

9. MacMahon H, Austin JH, Gamsu G, Herold CJ, Jett JR, Naidich NP, et al. Guidelines for management of small pulmonary nodules detected on CT scans: A statement from the Fleischner Society. Radiology 2005; 237(2):395-400.

10. Naidich DP, Bankier AA, MacMahon H, Schaefer-Prokop $\mathrm{CM}$, Pistolesi M, Goo JM, et al. Recommendations for the management of subsolid pulmonary nodules detected at CT: A statement from the Fleischner Society. Radiology 2013; 266(1):304-17.

11. Gurney JW. Determining the likelihood of malignancy in solitary pulmonary nodules with Bayesian analysis: I. Theory. Radiology 1993; 186:405-13.

12. Swensen SJ, Silverstein MD, Ilstrup DM, Schleck $C D$, Edell ES. The probability of malignancy in solitary pulmonary nodules. Application to small radiologically indeterminate nodules. Arch Intern Med 1997; 157(8):849-55.

13. Dewan NA, Shehan CJ, Reeb SD, Gobar LS, Scott WJ, Ryschon K. Likelihood of malignancy in a solitary pulmonary nodule: Comparison of Bayesian analysis and results of FDG-PET scan. Chest 1997; 112:41622.

14. Soubani AO. The evaluation and management of the solitary pulmonary nodule. Postgrad Med J 2008; 84(995):459-66.

15. Bach PB, Silvestri GA, Hanger M. Screening for lung cancer: ACCP evidence-based clinical practice guidelines. Chest 2007; 132(3):69-77.
16. McWilliams A, Tammemagi MC, Mayo JR, Roberts H, Liu G, Soghrati K, et al. Probability of cancer in pulmonary nodules detected on first screening $\mathrm{CT}$. $\mathrm{N}$ Engl J Med 2013; 369(10):910-9.

17. Wiener RS, Gould MK, Woloshin S, Schwartz LM, Clark JA. What do you mean, a spot? A qualitative analysis of patients' reactions to discussions with their physicians about pulmonary nodules. Chest 2013; 143(3):672-7.

18. Soardi GA, Perandini S, Motton M, Montemezzi $\mathrm{S}$. Assessing probability of malignancy in solid solitary pulmonary nodules with a new Bayesian calculator: Improving diagnostic accuracy by means of expanded and updated features. Eur Radiol 2015; 25(1): 155-162.

19. Travis WD, Brambilla E, Noguchi M, Nicholson $A G$, Geisinger KR, Yatabe $Y$, et al. International association for the study of lung cancer/American thoracic society/European respiratory society international multidisciplinary classification of lung adenocarcinoma. J Thorac Oncol 2011; 6(2):244-85.

20. Gohagan J, Marcus P, Fagerstrom R, Pinsky P, Kramer $B$, Prorok $P$. Writing committee, lung screening study research group. Baseline findings of a randomized feasibility trial of lung cancer screening with spiral CT scan vs chest radiograph: the Lung Screening Study of the National Cancer Institute. Chest 2004; 126:114-21.

21. Swensen SJ, Viggiano RW, Midthun DE, Müller $\mathrm{NL}$, Sherrick A, Yamashita $K$, et al. Lung nodule enhancement at CT: Multicenter study. Radiology 2000; 214(1):73-80.

22. Thomas JW, Staerkel GA, Whitman GJ. Pulmonary hamartoma. Am J Roentgenol 1999; 172: 1643.

23. Patel VK, Naik SK, Naidich DP, Travis WD, Weingarten $\mathrm{JA}$, Lazzaro $\mathrm{R}$, et al. A practical algorithmic approach to the diagnosis and management of solitary pulmonary nodules: Part 1: radiologic characteristics and imaging modalities. Chest 2013; 143 (3):825-39. 


\begin{tabular}{|l|l|l|}
\hline \multicolumn{3}{|c}{ AUTHORSHIP AND CONTRIBUTION DECLARATION } \\
\hline Sr. \# & \multicolumn{1}{|c|}{ Author(s) Full Name } & Contribution to the paper \\
\hline 1 & Oral Mentes & Study design, Data collection. \\
\hline 2 & Sema Avci & Editing, Writing. \\
\hline 3 & Suzan Bahadır & Study design. \\
\hline 4 & Meric Unver & Writing, Analysis. \\
\hline 6 & Feza Ugurman & Data collection. \\
\hline
\end{tabular}

